\title{
On Power Spectrum Optimization by Iterative Function Evaluation
}

\author{
Hayssam Dahrouj ${ }^{\dagger}$, Wei $\mathrm{Yu}^{\dagger}$, and Taiwen Tang ${ }^{\ddagger}$ \\ ${ }^{\dagger}$ Electrical and Computer Engineering Dept., University of Toronto, Canada \\ ${ }^{\ddagger}$ BlinQ Networks Inc., Kanata, Ontario, Canada \\ Emails: \{hayssam,weiyu\}@comm.utoronto.ca, eric.tang@blinqnetworks.com
}

\begin{abstract}
The problem of optimally adjusting the power spectral density level, referred as power spectrum optimization, is a well-known and difficult nonconvex optimization problem. This paper investigates a class of local optimization methods based on a technique called iterative function evaluation. This idea, first proposed in our previous work, is based on the manipulation of the first order condition of the optimization problem into an algorithm. This paper shows that these manipulations are not unique, and proposes a new iterative-function-evaluationbased method for power spectrum optimization. In addition, this paper proposes methods based on the average channel gains for an orthogonal frequency division multiple access network. Finally, the performance of the proposed methods is evaluated in a wireless backhaul application.
\end{abstract}

\section{INTRODUCTION}

Power spectrum optimization refers to the optimization of transmit powers in a multiuser environment for the purpose of managing mutual interference. Because of the mutual coupling between the power terms which induces nonconvexity, finding the global optimality of the power spectrum optimization problem is understood to be a difficult problem.

Like many existing approaches to this problem, this paper focuses only on local optimization methods, but aims to identify algorithms with fast convergence. In particular, we adopt an approach based on iterative function evaluation methods (IFEMs), first proposed in [1], in which an iterative algorithm is derived based on the manipulation of the first order condition of the optimization problem.

This paper shows that such manipulation is not unique; different manipulations lead to different iterative power optimization algorithms. But one also has to be careful in choosing the sensible manipulations in order to ensure convergence. While our previous work proposed an IFEM method that has the flavor of the SCALE algorithm [2] for power optimization, this paper proposes a different IFEM method that has the flavor of iterative water-filling [3].

The proposed method is computationally fast. It can be implemented in a distributed fashion, and does not require step size choices, as traditional subgradient or Newton's methods in optimization do. Finally, this paper also proposes numerical power adaptation algorithms based on the average channel gains, and evaluates their performance on a wireless backhaul application.

\section{System Model And Problem Formulation}

\section{A. System Model}

This paper considers a wireless backhaul network with $L$ access nodes (ANs) and $K$ remote terminals (RTs) per AN, as in [1], with single antenna at both the ANs and RTs. The paper uses an orthogonal frequency division multiple access (OFDMA) scheme with $N$ subcarriers to separate the RTs belonging to each $\mathrm{AN}$. For each $\mathrm{AN} l$, only one $\mathrm{RT} k$ is active at each frequency tone $n$. More formally, the scheduled RT of the $l$ th $\mathrm{AN}$ at the $n$th tone is denoted by $k=f(l, n)$. Similarly, $k^{\prime}=f(j, n)$ denotes the scheduled RT of the $j$ th AN at the $n$th tone. Let $x_{l}^{n}$ be the complex scalar denoting the information signal for the $k$ th $\mathrm{RT}$. The received signal at the $k$ th $\mathrm{RT}$ can be written as:

$$
y_{l}^{n}=h_{l l k}^{n} x_{l}^{n}+\sum_{j \neq l} h_{j l k}^{n} x_{j}^{n}+z_{l}^{n}
$$

where $h_{j l k}^{n} \in \mathbb{C}$ is the channel from the $j$ th $\mathrm{AN}$ to the $k$ th $\mathrm{RT}$, and $z_{l}^{n}$ is the additive white Gaussian noise with variance $\sigma^{2} / 2$ on each of its real and imaginary components.

\section{B. Problem Formulation}

Similar to our previous work [1], this paper adopts a network utility maximization framework with an objective of maximizing the proportionally fair log utility function of the long-term average rate: $\sum_{l, k} \log \left(\bar{R}_{l k}\right)$, where $\bar{R}_{l k}$ is the long term average rate of the $k$ th $\mathrm{RT}$ of the $l$ th AN. The problem consequently boils down to a per-tone weighted ratesum maximization problem:

$$
\begin{array}{ll}
\max & \sum_{l} w_{l k} r_{l k}^{n} \\
\text { s.t. } & 0 \leq P_{l}^{n} \leq S^{\max }
\end{array}
$$

where

$$
r_{l k}^{n}=\log \left(1+\frac{P_{l}^{n}\left|h_{l l k}^{n}\right|^{2}}{\Gamma\left(\sigma^{2}+\sum_{j \neq l} P_{j}^{n}\left|h_{j l k}^{n}\right|^{2}\right)}\right)
$$

is the instantaneous rate of the scheduled $k$ th $\mathrm{RT}$ for the $l$ th AN at the $n$th tone, $S^{\max }$ is the maximum power constraint imposed on each $\mathrm{AN}$ at each tone, the weights come from the scheduling objective (e.g. $w_{l k}=\frac{1}{\bar{R}_{l k}}$ for proportional fairness), and where the maximization is over the set of powers $P_{l}^{n}$. 


\section{Algorithms}

Due to the nonconvexity of the problem (2), this paper, like [1] and many previous works, aims at local optimal solutions. The proposed method is based on the observation that the gradient of the objective function is zero at a local optimum, and that the manipulations of the optimality condition can lead to power update algorithms. These algorithms are called iterative function evaluation methods (IFEMs).

\section{A. IFEMS}

The objective function of the problem (2), which is optimized over the set of power on a tone-by-tone basis, can be written as:

$R\left(\left\{P_{i}^{n}\right\}_{i=1}^{L}\right)=\sum_{l} w_{l k} \log \left(1+\frac{P_{l}^{n}\left|h_{l l k}^{n}\right|^{2}}{\Gamma\left(\sigma^{2}+\sum_{j \neq l} P_{j}^{n}\left|h_{j l k}^{n}\right|^{2}\right)}\right)$

We begin by taking the derivative of the objective function $R$ with respect to $P_{l}^{n}$ :

$$
\begin{aligned}
\frac{\partial R}{\partial P_{l}^{n}=} & w_{l k} \frac{\partial r_{l k}^{n}}{\partial P_{l}^{n}}+\sum_{j \neq l} w_{j k^{\prime}} \frac{\partial r_{j k^{\prime}}^{n}}{\partial P_{l}^{n}} \\
= & \frac{w_{l k}}{P_{l}^{n}}\left(\frac{\operatorname{SINR}_{l}^{n}}{1+\operatorname{SINR}_{l}^{n}}\right)- \\
& \sum_{j \neq l} w_{j k^{\prime}} \frac{\left|h_{l j k^{\prime}}^{n}\right|^{2}}{\sigma^{2}+\sum_{i \neq j} P_{i}^{n}\left|h_{i j k^{\prime}}^{n}\right|^{2}} \frac{\operatorname{SINR}_{j}^{n}}{1+\operatorname{SINR}_{j}^{n}}
\end{aligned}
$$

where $\operatorname{SINR}_{j}^{n}$ is defined as:

$$
\operatorname{SINR}_{j}^{n}=\frac{P_{j}^{n}\left|h_{j j k^{\prime}}^{n}\right|^{2}}{\Gamma\left(\sigma^{2}+\sum_{i \neq j} P_{i}^{n}\left|h_{i j k^{\prime}}^{n}\right|^{2}\right)},
$$

and $k=f(l, n)$ and $k^{\prime}=f(j, n)$ are the scheduled RTs of the $l$ th $\mathrm{AN}$ and the $j$ th $\mathrm{AN}$ respectively at the $n$th tone. A local optimal solution must be such that the above gradient is zero. The key idea of IFEM is that by setting the above gradient to zero and by manipulating the optimality condition, one can obtain algorithms for optimizing power.

One possible manipulation of the optimality condition gives the following equation:

$$
P_{l}^{n}=\frac{w_{l k} \frac{\operatorname{SINR}_{l}^{n}}{1+\operatorname{SINR}_{l}^{n}}}{\sum_{j \neq l} w_{j k^{\prime}} \frac{\left|h_{l j k^{\prime}}^{n}\right|^{2}}{\sigma^{2}+\sum_{i \neq j} P_{i}^{n}\left|h_{i j k^{\prime}}^{n}\right|^{2}} \frac{\operatorname{SINR}_{j}^{n}}{1+\operatorname{SINR}_{j}^{n}}} .
$$

This leads to an algorithm called full-IFEM in [1]. In this paper, we call it IFEM-1 for the sake of comparing it with the new algorithms.

The main point of this paper is that the manipulation used in deriving IFEM-1 is not unique. In fact, different manipulations of the optimality condition can lead to different algorithms. For example, starting again by setting the gradient (4) to zero, we can write

$$
P_{l}^{n}\left(1+\frac{1}{\operatorname{SINR}_{l}^{n}}\right)=\frac{w_{l k}}{\sum_{j \neq l} \frac{w_{j k^{\prime}}\left|h_{l j k^{\prime}}^{n}\right|^{2}}{\sigma^{2}+\sum_{i \neq j} P_{i}^{n} \mid h_{i j k^{\prime}}^{n}} \frac{\operatorname{SINR}_{j}^{n}}{1+\operatorname{SINR}_{j}^{n}}}
$$

Thus,

$$
P_{l}^{n}=\frac{w_{l k}}{\sum_{j \neq l} w_{j k^{\prime}} \frac{\left|h_{l j k^{\prime}}^{n}\right|^{2}}{\sigma^{2}+\sum_{i \neq j} P_{i}^{n}\left|h_{i j k^{\prime}}^{n}\right|^{2}} \frac{\operatorname{SINR}_{j}^{n}}{1+\operatorname{SINR}_{j}^{n}}}-\frac{P_{l}^{n}}{\operatorname{SINR}_{l}^{n}}
$$

Again, we can interpret the above equation as an iterative algorithm, i.e., one can compute the terms on the right-hand side, and update the new power allocation according to (8). We name the resulting new algorithm IFEM-2. More formally, in IFEM-2 the power level of every AN at every tone, $P_{l}^{n}$, is updated from step $t$ to $t+1$ according to

$$
P_{l}^{n}(t+1)=\left[\frac{w_{l k}}{\sum_{j \neq l} \tau_{j l}^{n}(t)}-\frac{P_{l}^{n}(t)}{\operatorname{SINR}_{l}^{n}(t)}\right]_{0}^{S^{\max }}
$$

where the power constraint is again taken into account using the projection step $[.]_{0}^{S^{\max }}$.

This strategy of deriving a fixed-point equation for power optimization by isolating the power term $P_{l}^{n}$ on one part of the equation is by no means limited to the above methods, i.e. IFEM-1 and IFEM-2. For example, one can rearrange the terms in (4) in a different way to get the following relation:

$$
P_{l}^{n}=\operatorname{SINR}_{l}^{n}\left(\frac{w_{l k}}{\sum_{j \neq l} \frac{w_{j k^{\prime}}\left|h_{l j k^{\prime}}^{n}\right|^{2}}{\sigma^{2}+\sum_{i \neq j} P_{i}^{n}\left|h_{i j k^{\prime}}^{n}\right|^{2}} \frac{\operatorname{SINR}_{j}^{n}}{1+\operatorname{SINR}_{j}^{n}}}-P_{l}^{n}\right),
$$

which we call IFEM-3 in this paper. But interestingly, although both IFEM-1 and IFEM-2 are excellent algorithms, IFEM3 is not. It turns out that the iterative function evaluation method resulting from this above manipulation (10) does not necessarily converge. The reason behind such differences is not well understood. It nevertheless serves to illustrate that one has to be careful in coming up with different algorithms.

\section{B. High-SINR IFEM (HSIFEM) and $\theta$-IFEM-2}

The convergence of both IFEM-1 and IFEM-2 is not easy to establish in full generality, due to the tight coupling of the interference terms in their respective update equations. In [1], two-algorithms called $\theta$-IFEM-1 (called $\theta$-IFEM in [1]) and IFEM are proposed to approximate IFEM-1. The advantage of such approximation is that the convergence of the two algorithms IFEM and $\theta$-IFEM- 1 can be proven based on the standard function properties introduced in [4]. While IFEM is a high-SINR approximation of IFEM-1, $\theta$-IFEM-1 replaces the per-iteration SINRs in the IFEM-1 update equation with fixed values of SINRs calculated under the initial maximum power transmission strategy.

Similar approaches can be applied to IFEM-2. First, note that at a high SINR, both IFEM-1 and IFEM-2 reduce to the same update equation. This high-SINR approximation, which is the same as IFEM in [1], has the following power update equation:

$P_{l}^{n}(t+1)=\left[\frac{w_{l k}}{\sum_{j \neq l} w_{j k^{\prime}} \frac{\left|h^{n}{ }_{l j k^{\prime}}\right|^{2}}{\sigma^{2}+\sum_{i \neq j} P_{i}^{n}(t)\left|h^{n}{ }_{i j k^{\prime}}\right|^{2}}}\right]_{0}^{S^{\max }}$ 
We call this algorithm High-SINR IFEM (HSIFEM).

Moreover, following the same idea used in deriving $\theta$ IFEM-1 from IFEM-1, a similar method can be applied to replace the per-iteration SINRs in the IFEM-2 update equation with the values of SINRs calculated under the initial maximum power transmission strategy. However, it turns out that this method does not necessarily converge. But for the sake of completeness, we write down the $\theta$-IFEM- 2 algorithm, which updates the power level $P_{l}^{n}$ according to:

$P_{l}^{n}(t+1)=\left[\frac{w_{l k}}{\sum_{j \neq l} \frac{w_{j k^{\prime}}\left|h^{n} l j k^{\prime}\right|^{2}}{\sigma^{2}+\sum_{i \neq j} P_{i}^{n}(t)\left|h^{n}{ }_{i j k^{\prime}}\right|^{2}} \theta_{j}^{n}}-\frac{P_{l}^{n}(t)}{\frac{\theta_{l}^{n}}{1-\theta_{l}^{n}}}\right]_{0}^{S^{\max }}$

where $\theta_{l}^{n}=\frac{\widetilde{\operatorname{SINR}}_{l}^{n}}{1+\widetilde{\operatorname{SINR}}_{l}^{n}}$, and $\widetilde{\operatorname{SINR}}_{l}^{n}$ is the fixed SINR calculated from the maximum power transmission strategy, i.e.

$$
{\widetilde{\operatorname{SINR}_{l}}}_{l}^{n}=\frac{S^{\text {max }}\left|h_{l l k}^{n}\right|^{2}}{\Gamma\left(\sigma^{2}+\sum_{j \neq l} S^{\text {max }}\left|h_{j l k}^{n}\right|^{2}\right)}
$$

\section{Methods Based on Average Channel Gains:}

All the methods above are based on per-tone channel measurements. Those measurements are typically provided to either a central server for further centralized processing, or to each of the several access nodes for distributed processing. In practice, to minimize the overhead of such exchange of information, the measurements can alternatively provide the frequency domain average channel gains. The proposed methods above can make use of the average channel gains instead. In the simulations section, we subsequently quantify the performance of these "average gains methods" in a typical wireless backhaul network. These methods are of particular interest when the delay spread of the wireless propagation environment is limited.

Define the average channel gain $\left|\bar{h}_{j l k}\right|^{2}$ as:

$$
\left|\bar{h}_{j l k}\right|^{2}=\frac{1}{N} \sum_{n=1}^{N}\left|h_{j l k}^{n}\right|^{2}, \quad \forall(j, l, k)
$$

All the methods presented earlier become "Average Gain methods (AG method)" by substituting the average gains in the equations of the corresponding method. For example, IFEM-2 becomes AG IFEM-2. To evaluate the performance of the methods in this paper, we compare them with fullblown Newton's method (NM) proposed in [5]. The update equations of NM and its high-SINR approximation (HSNM) can be found in [1].

\section{Distributed Implementation:}

The above methods in this paper can be put together under a generic power update equation:

$$
P_{l}^{n}(t+1)=\vartheta\left(\tau_{j l}^{n}(t), P_{l}^{n}(t), \operatorname{SINR}_{l}^{n}(t)\right)
$$

Typically, $P_{l}^{n}$ is known and $\operatorname{SINR}_{l}^{n}$ can be easily measured at each AN. A distributed implementation of power allocation (15) is, therefore, possible if ANs exchange the variables $\tau_{j l}^{n}$. The terms $\tau_{j l}^{n}$ typically have the interpretation of being the interference prices.

\begin{tabular}{|l|c|c|}
\hline Sum Rate in bps/Hz & $d_{1}=0.5 \mathrm{~km}$ & $d_{1}=1 \mathrm{~km}$ \\
\hline \hline HSIFEM & 60.68 & 91.33 \\
\hline AG HSIFEM & 60.67 & 91.33 \\
\hline HSNM & 60.68 & 91.33 \\
\hline AG HSNM & 60.67 & 91.33 \\
\hline IFEM-1 & 62.61 & 91.58 \\
\hline AG IFEM-1 & 62.59 & 91.57 \\
\hline IFEM-2 & 62.61 & 91.58 \\
\hline AG IFEM-2 & 62.59 & 91.57 \\
\hline NM & 62.61 & 91.58 \\
\hline AG NM & 62.59 & 91.57 \\
\hline Max Power Method & 53.01 & 86.22 \\
\hline \hline IFEM-2 Gain & $18.1 \%$ & $6.2 \%$ \\
\hline
\end{tabular}

TABLE I

Methods BeHAVIOR OVER 7 ANs, 4 RTS PER AN. $d_{1}$ IS THE AN-TO-AN DISTANCE. AN-TO-RT DISTANCE IS $150 \mathrm{M}$.

\begin{tabular}{|l|c|c|}
\hline Sum Rate in bps/Hz & Cell-edge $\left(d_{2}=333 \mathrm{~m}\right)$ & Cell-center $\left(d_{2}=125 \mathrm{~m}\right)$ \\
\hline \hline HSIFEM & 34.84 & 78.39 \\
\hline AG HSIFEM & 34.83 & 78.38 \\
\hline HSNM & 34.84 & 78.39 \\
\hline AG HSNM & 34.83 & 78.38 \\
\hline IFEM-1 & 41.11 & 78.77 \\
\hline AG IFEM-1 & 41.09 & 78.76 \\
\hline IFEM-2 & 41.11 & 78.77 \\
\hline AG IFEM-2 & 41.09 & 78.76 \\
\hline NM & 41.11 & 78.77 \\
\hline AG NM & 41.09 & 78.76 \\
\hline Max Power Method & 30.54 & 71.91 \\
\hline \hline IFEM-2 Gain & $34.6 \%$ & $9.5 \%$ \\
\hline
\end{tabular}

TABLE II

METHOdS BEHAVIOR OVER 7 ANS, 4 RTS PER AN. AN-TO-AN DISTANCE IS $0.5 \mathrm{KM}$.

\section{Simulations}

The performance of the proposed methods is evaluated using a wireless backhaul network comprising 7 ANs, and 4 RTs per $\mathrm{AN}$, over $10 \mathrm{MHz}$ bandwidth. It assumes an OFDMA system with 1024 subcarriers, where only one RT is active at each frequency tone. A distance-dependent pathloss model $128.1+37.6 \log _{10}(d)$, where $k$ is in $\mathrm{km}$, and a multipath delay profile corresponding to SUI-3 Terrain type B are adopted. To study the performance of the proposed methods for various topologies, the AN-to-AN distance $d_{1}$ and the AN-to-RT distance $d_{2}$ vary throughout the simulations. To compare the sum rate of the various methods, the weighting factors $w_{l k}$ in problem (2) are set to $1 \forall(l, k)$.

Tables I and II show the sum-rate performance of the proposed methods for different network topologies. Tables I and II show that IFEM-1, IFEM-2, and NM all have similar performance over all topologies, i.e., they all converge to a local optimal solution of the original nonconvex optimization problem. In addition, HSIFEM and HSNM, which are derived under the high SINR approximation, also always have the same performance. For cell-center RTs, e.g. the case of $d_{1}=1 \mathrm{~km}, d_{2}=150 \mathrm{~m}$ in Table I and the case of $d_{1}=0.5 \mathrm{~km}$, $d_{2}=125 \mathrm{~m}$ in Table II, the high SINR approximation is valid. In this case, HSIFEM, HSNM, IFEM-1,IFEM-2 and NM all 


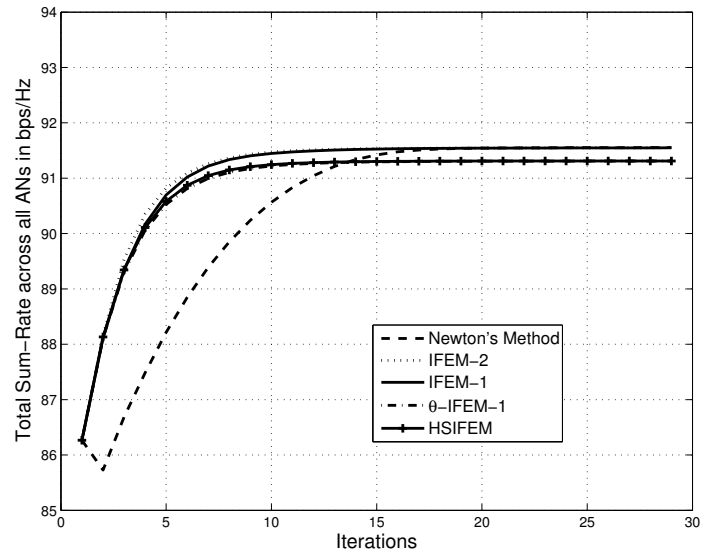

Fig. 1. Sum-rate in $\mathrm{bps} / \mathrm{Hz}$ versus the number of iterations, over $7 \mathrm{ANs}$, 4 RTs per AN. AN-to-AN distance is $1 \mathrm{~km}$. AN-to-RT distance is $150 \mathrm{~m}$. It shows the convergence of the different methods at high SINR.

have similar performance. But when the SINR level is not sufficiently high as it is the case for cell-edge RTs, HSIFEM and HSNM become inferior to IFEM-1, IFEM-2 and NM. Note that all the proposed algorithms remain superior to the maximum power method strategy, for all network topologies.

Tables I and II also show that the methods that rely on the frequency domain average channel gain have a very similar performance to the methods that are based on the per-tone channel gain measurements. This is due to the fact that a typical fixed wireless backhaul channel is relatively flat fading. Our simulation assumes an SUI-3 model with Terrain type B, in which the delay profile has three taps; the k-factors of the three taps are 3, 0, and 0 ; the relative taps delays are $0,0.4$, and 0.9 in microseconds.

The convergence performance of the various proposed algorithms is shown in Figs. 1 and 2. The step size of Newton's method is fixed to 1 , so as to allow a fair comparison with the IFEMs. Fig. 1 corresponds to a high SINR situation. In this situation, the Newton's method has a poor performance as compared to the IFEMs, IFEM-2 shows the overall faster convergence. In a low SINR situation, however, as it is the case in Fig. 2, the convergence of the Newton's method is faster than both IFEM-1 and IFEM-2.

Finally, Fig. 3 illustrates the convergence behavior of IFEM3 and $\theta$-IFEM-2. Unlike the other proposed methods in the paper, these two algorithms show an oscillatory behavior. IFEM3 particularly shows an inferior performance as compared to all other methods.

\section{CONCLUSION}

Dynamic power spectrum optimization is expected to play a major role in improving the performance of future wireless networks. This paper proposes novel methods for power management. The methods are based on either the frequency domain channel gains, or the frequency domain channel average gains. The methods are computationally feasible and can be implemented in a distributed fashion. They do not require step

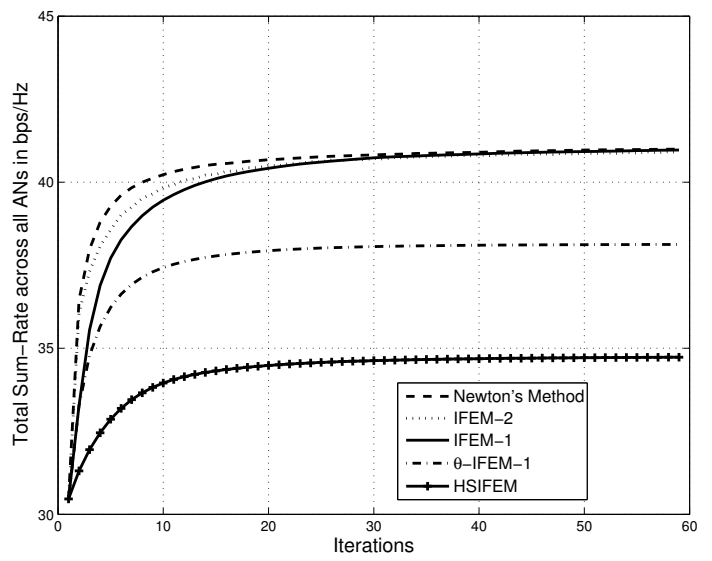

Fig. 2. Sum-rate in bps/Hz versus the number of iterations, over 7 ANs, 4 RTs per AN. AN-to-AN distance is $0.5 \mathrm{~km}$. AN-to-RT distance is $333 \mathrm{~m}$. It shows the convergence of the different methods at low SINR.

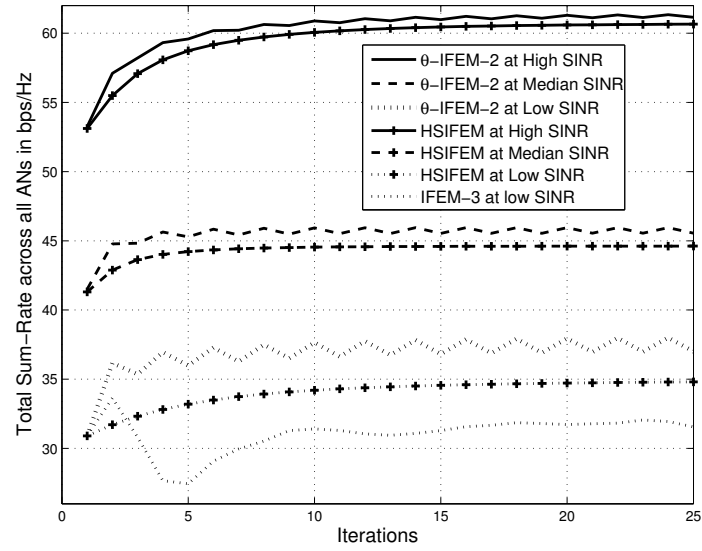

Fig. 3. Sum-rate in bps/Hz versus the number of iterations, over 7 ANs, 4 RTs per AN, for different SINR levels. It shows the oscillatory convergence behavior of $\theta$-IFEM-2 and IFEM-3.

size choices, as traditional subgradient or Newton's methods in optimization do. They show a significant performance improvement as compared to the maximum power transmission strategy.

\section{REFERENCES}

[1] H. Dahrouj, W. Yu, T. Tang, and S. Beaudin, "Power spectrum optimization for interference mitigation via iterative function evaluation," in Proc. First Workshop on Distributed Antenna Systems for Broadband Mobile Communications IEEE Global Telecommun. Conf. (Globecom), Houston, U.S.A., Dec. 2011.

[2] J. Papandriopoulos and J. S. Evans, "Low-complexity distributed algorithms for spectrum balancing in multi-user DSL networks," in Proc. IEEE Inter. Conf. Commun. (ICC), Istambul, Turkey, Jun. 2006.

[3] W. Yu, "Multiuser water-filling in the presence of crosstalk," in Inform. Theory and Appl. Workshop, San Diego, U.S.A., 2007.

[4] R. Yates, "A framework for uplink power control in cellular radio systems," IEEE J. Sel. Areas Commun., vol. 13, no. 7, pp. 1341-1347, Sep. 1995.

[5] W. Yu, T. Kwon, and C. Shin, "Joint scheduling and dynamic power spectrum optimization for wireless multicell networks," in Conference on Information Science and Systems (CISS), Princeton, NJ, Mar. 2010. 